\title{
CULTURA SE FAZ BRINCANDO: UM ESTUDO SOBRE AS BRINCADEIRAS DE CRIANÇAS NA CULTURA GAVIÃO
}

\author{
Luciana de Oliveira Dias ${ }^{1}$ \\ Leonice Cardoso Gavião ${ }^{2}$
}

\section{RESUMO}

O presente texto resulta da experiência de realização de um estágio supervisionado, do Comitê Gavião-Timbira, na Escola Indígena Pýr Creh' Creht, localizada na Aldeia Riachinho, no Maranhão. Um estudo das brincadeiras na cultura gavião foi o tema contextual escolhido para a realização das aulas. O objetivo com a seleção da temática foi trabalhar com estudantes e demais moradores da aldeia sobre alguns processos de interação sociocultural, com destaque para a importância das brincadeiras na cultura gavião. Como resultado, alcançamos reflexões que evidenciam que, além de momentos de descontração, alegria e lazer, as brincadeiras são também locus de ensino, aprendizagem e revitalização da própria cultura de um povo.

Palavras-chave: Cultura. Brincadeiras. Povo Gavião.

1 Antropóloga, pós-doutora em Direitos Humanos e Interculturalidades pela Universidade de Brasília - UnB. Professora do Núcleo Takinahakỹ de Formação Superior de Professores Indígenas e do Programa de Mestrado Interdisciplinar em Direitos Humanos, da Universidade Federal de Goiás (UFG). Coordenadora do Coletivo Rosa Parks - UFG. Goiânia, GO, Brasil. E-mail: professoralucianadias@gmail.com.

2 Licenciada em Educação Intercultural, com habilitação em Ciências da Natureza, pela UFG. Professora da Educação Básica. Amarante, MA, Brasil. E-mail: leonicecgaviao@gmail.com. 


\section{ABSTRACT}

This text presents the experience of a supervised internship, of the GaviãoTimbira Committee, at the Pýr Creh' Creht Indigenous School, located in Aldeia Riachinho, in Maranhão, Brazil. The study of play in gaviãoculture was the theme chosen for the classes. The objective was to work with students and other members of the community on some processes of sociocultural interaction, with emphasis on the importance of play in the gavião culture. The result was reflections that show that, in addition to moments of relaxation, joy andrecreation, the games are also locus of teaching, learning and revitalization of the own culture of a people.

Key Words: Culture. Games.Gavião People.

\section{Introdução}

O texto ora apresentado é um resultado da experiência de Estágio Supervisionado desenvolvido pela professora Leonice Cardoso Gavião, sob a supervisão e acompanhamento de sua orientadora, a professora Luciana de Oliveira Dias. As aulas aconteceram na Aldeia Riachinho, localizada na Terra Indígena Governador, do Povo Gavião, no estado do Maranhão. Destacamos que as aulas, inicialmente propostas para as crianças da educação infantil e do $9^{\circ}$ ano, extrapolaram os limites da Escola Indígena Pýr Creh' Creh, acontecendo no pátio da aldeia. E, expandiram também os limites da relação docente/discente ao assegurarem a participação da professora, de estudantes e de todos os outros membros da comunidade que se sentiram estimulados, depois de convidados, a realizarem as aulas sobre brincadeiras na cultura do Povo Gavião -Pyhcopcati ji.

No âmbito da Educação Intercultural Indígena, da Universidade Federal de Goiás - UFG, o estágio supervisionado, além de preparar para a docência, constitui-se também como um importante instrumento de formação que tem levado todos os sujeitos envolvidos a profundas reflexões acerca da própria prática pedagógica, do cotidiano da sala de aula e do processo de ensino e aprendizagem, e, da relação entre teoria e prática. Neste sentido, o desenvolvimento de um estágio tem permitido o alcance de uma lucidez no que se refere à produção e reprodução da prática pedagógica e da vida vivida em suas dimensões culturais, políticas, filosóficas, linguísticas e ideológicas. 
Com o objetivo de atender a todas essas demandas de ensino e aprendizagem que partem tanto da Educação Intercultural Indígena, da UFG, quanto da Aldeia Riachinho foi construída a proposta de trabalhar com o tema contextual "brincadeiras na cultura Gavião". Para a realização da proposta buscamos em um primeiro momento a concretização de um material didático adequado e coerente com a proposta temática. A pesquisa emergiu como caminho satisfatoriamente transitável e geradora de elementos que compusessem o cabedal que daria início às práticas pedagógicas intencionadas para aquele estágio. Concordamos com Paulo Freire (2002) quando o mesmo afirma que

Não há ensino sem pesquisa e pesquisa sem ensino. Esses que-fazeres se encontram um no corpo do outro. Enquanto ensino continuo buscando, reprocurando. Ensino porque busco, porque indaguei, porque indago e me indago. Pesquiso para constatar, constatando, intervenho, intervindo educo e me educo. Pesquiso para conhecer o que ainda não conheço e comunicar ou anunciar a novidade (FREIRE, 2002, p. 32).

Investidas pelo desejo de busca e de indagação, enquanto buscávamos e indagávamos a nós mesmas, procuramos comunicar e anunciar a novidade a partir da realização de uma entrevista, em profundidade, a uma anciã gavião, profunda conhecedora dos hábitos e costumes do Povo Gavião - Pyhcopcati ji. Nos últimos dias do mês de dezembro de 2016, foi realizada a entrevista com a senhora $A$ 'xu - Maria Amélia, que vive na Aldeia Monte Alegre. A entrevista, que foi gravada e depois transcrita, trouxe incontáveis histórias de brincadeiras que agradam crianças e adultos gavião, algumas completamente vivas e que fazem parte do cotidiano de qualquer aldeia gavião, outras que caíram no esquecimento. Estas últimas merecem um trabalho à parte, considerando que seguem vivas somente na memória de dona $A^{\prime} x u$ e talvez algum dia escrevamos especificamente sobre essas memórias resistentes, portanto existentes.

Para a realização do estágio supervisionado, que segue agora inspirando a escrita deste artigo, selecionamos cinco brincadeiras dentre todas aquelas que foram rememoradas pela anciã entrevistada e que foram, durante a realização das aulas, transformadas em oficinas que aconteceram no barracão que fica no pátio central da Aldeia Riachinho. As brincadeiras que foram selecionadas e sobre 
as quais discorremos no corpo deste texto, são: (a) a brincadeira de Capryynre, que chamamos em português de brincadeira de jabuti; (b) a brincadeira de $A$ 'cruu 'te, que recebeu o nome de brincadeira do cipó, em português; (c) a brincadeira de $P y ́ r$ Cahýc, que não conseguimos traduzir para o português; (d) a brincadeira de Pryyhre 'proxý, que tem como equivalente na língua portuguesa: a brincadeira do tatu; e, (e) Cahowcra'crat, que decidimos manter seu nome somente na língua gavião por dificuldades em fazer uma tradução.

Foto 1 - Anciã durante a oficina sobre brincadeiras na cultura Gavião.

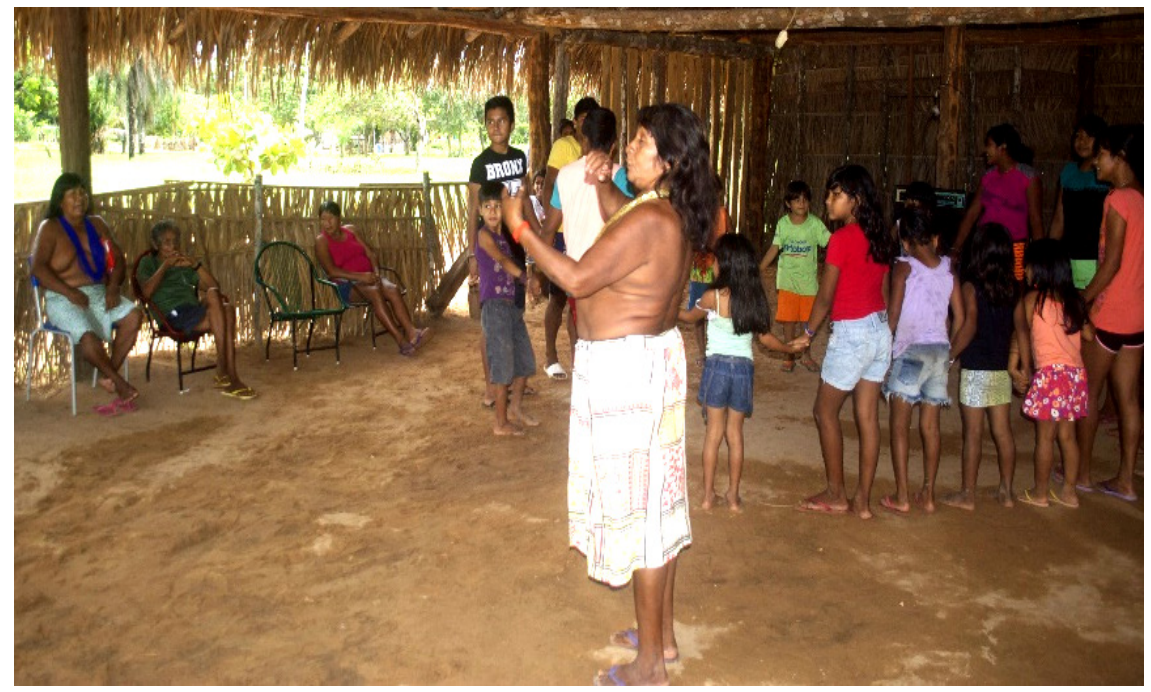

Fonte: As autoras, março de 2017.

Um primeiro aprendizado, imediatamente revelado a nós, foi o de que são múltiplas e criativas as possibilidades de sociabilidade e interação sociocultural, por meio de brincadeiras, entre o povo gavião. Os espaços educacionais podem ser compreendidos como provocadores de complexos processos de significação (GÓES, 1997), nos quais a própria cultura é revitalizada com a aquisição de sentido e significado, o que pode se dar, inclusive, por meio das brincadeiras. Há uma potência e capacidade de envolvimento de todos os sujeitos nesses processos de significação, sendo que na experiência do estágio supervisionado crianças, adolescentes, adultos e anciãos, estudantes ou não, foram partícipes altamente ativos na realização das aulas. Aulas que extrapolaram seu objetivo inicial, já que imprimiram no 
final das atividades um sentimento parecido com algo como "tenho orgulho de pertencer ao Povo Gavião".

\section{Brincando também se constroem vidas}

A interação com o meio sociocultural no qual os sujeitos estão inseridos promove a capacidade individual e grupal de crescimento e desenvolvimento, bem como tem o potencial de reforçar os laços que suturam o sujeito à cultura. A cultura, que se manifesta sobremaneira em instantes de interação e sociabilidade, "condiciona a visão de mundo" dos indivíduos, como enfatiza Roque de Barros Laraia (1986, p. 67). Partindo desse pressuposto, as brincadeiras na cultura gavião compreendem um modo de ver o mundo gavião, bem como apreciações de ordem valorativa, capazes de imprimir significações nas ações e ativar processos de ensino e aprendizagem da própria cultura.

As brincadeiras além de propiciarem descontração e lazer apresentam também uma função simbólica e funcional, na qual as ideologias e os signos produzidos pela cultura são repassados, reafirmados, socializados e re-editados. Gilles Bougére (2001) destaca que as pessoas que se envolvem em uma brincadeira, entram em contato também com todos os signos produzidos pela cultura à qual pertencem. O que o autor nos demonstra é que há uma intrínseca relação entre brincadeira e cultura que fazem com que nossas maneiras de significar, de pensar e agir no mundo, são eminentemente dialéticas com relação aos grupos aos quais fazemos parte.

As cinco brincadeiras que foram selecionadas para compor este artigo lançam luz sobre formas de vida do Povo Gavião Pyhcopcati ji que são didaticamente ensinadas e aprendidas em contextos de educação e cultura. Para que mais aprofundadamente se compreenda o eixo teórico que estamos aqui defendendo, apresentamos a seguir de maneira detalhada, analítica e reflexiva o conjunto das brincadeiras que compuseram o estágio supervisionado da professora gavião.

A primeira brincadeira a ser destacada é a Capryynre, que pode ser chamada em português de brincadeira de jabuti. A Capryynre pode ser descrita como uma brincadeira marcada pelo divertimento geralmente noturno ou de final de dia. As crianças e jovens são incentivadas pelos anciãos e anciãs a iniciar a Capryynre. A pessoa que é escolhida para iniciar deve ser um pouco maior que 
as demais, deve ser "alguém mais fortinho", com idade aproximada entre 15 a 17 anos. Essa pessoa selecionada deve segurar-se em uma estaca de madeira firmada no chão. As outras pessoas que participam da brincadeira devem segurar os pés daquele que está deitado no chão e segurando firmemente a estaca de madeira. Uma fila se forma até que todos os participantes fiquem também deitados e puxando os pés daquele que está à frente, como se fosse a representação de um jabuti. O último da fila deve puxar com força as pernas daquele que está à frente até chegar no primeiro da fila. Quando chegar na pessoa que está no início da fila, segurando a estaca de madeira, todos os participantes da brincadeira puxam sua a perna até desprender.

Fotos 2 e 3 - Brincadeira Capryynre.
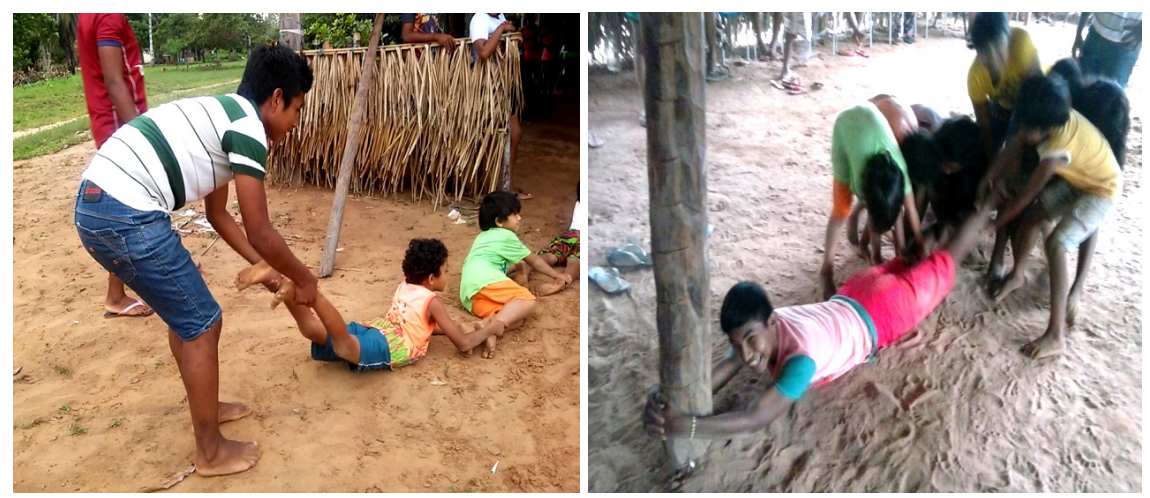

Fonte: As autoras, março de 2017.

Para que tenhamos um alcance maior da explicação desta brincadeira, apresentamos a seguir uma descrição na língua gavião: Caprynrejyycron te hararquicaêh'cryyrejôhmpêhxentpêh'nyhoowahe tyx ne no,quicaêh'cutme 'cwyajpente'nyhowahe'tyx neca me hêecwahquica me hapýyryyteapencut,quicajôhpêhxitme'tenycohcwah ne camecji ne came'cryntomo, ne me'cryynpato ne,êh'protpijôhmêh'cryyreno na wýrcato,ne ca me ta'nyêh'prot ne ca me capahquicaamreho ne Capryynrejýycron te hajyr.

Assim como a brincadeira de Capryynre, as aulas sobre ela foram bastante criativas. O aprendizado foi além das regras da própria brincadeira, já que muitas pessoas não conheciam o Capryynre e seus significados. Aprendemos a nos divertir coletivamente, mas aprendemos, sobretudo, a importância do respeito que deve sempre 
estar presente entre seres humanos e demais animais da natureza. A palavra Capryynre significa jabuti e o animal é imitado pelos integrantes da brincadeira, o que estampa, nesse instante, de lazer e aprendizado a importância de percepção da proximidade entre seres humanos e demais animais da natureza. O convívio harmônico e respeitoso entre todos os seres vivos é um valor para a cultura gavião e por meio da brincadeira Capryynre o respeito pela natureza é transmitido.

O equilíbrio entre todas as formas de vida existentes é uma busca constante para o Povo Gavião - Pyhcopcati ji. Em um estudo específico sobre o território gavião, a questão da preservação do território de maneira equilibrada foi profundamente discutida, reconhecendo a urgente necessidade de convício harmônico entre todas as formas de vida e chamando a atenção para o fato de que dentro dos limites territoriais gavião sempre é buscado um "[...] equilíbrio entre seres humanos e natureza, com o objetivo de proteger a terra das influências negativas externas, que, entre outras coisas, enfraquece a cultura, vulnerabiliza os costumes e prejudica o meio ambiente que é partilhado com toda a comunidade Gavião" (DIAS, 2017, p. 15-16).

A segunda brincadeira é a $A^{\prime}$ cruu'te, que pode ser traduzida sem prejuízo de sua compreensão como brincadeira do cipó, envolve todas as pessoas presentes, de qualquer sexo ou idade. Também é ensinada pelas anciãs e anciãos e para que ela aconteça também deve-se formar uma fila. Essa fila deve ser construída com umas pessoas segurando firme nas mãos de outras com seus braços cruzados. De mãos dadas, uns vão entrando debaixo do outro até que se chegue na primeira pessoa da fila, ao chegar no primeiro da fila. A partir daí, o último da fila começa a enrolar, sempre de mãos dadas e segurando firme para que as mãos não se soltem. Segue-se enrolando a fila e quando terminar de enrolar, a pessoa que estava puxando o movimento circular, começa a desenrolar. A crença é a de que aquele que soltar a mão do colega que está ao lado, antes que a brincadeira termine, perderá todos os seus dentes. 

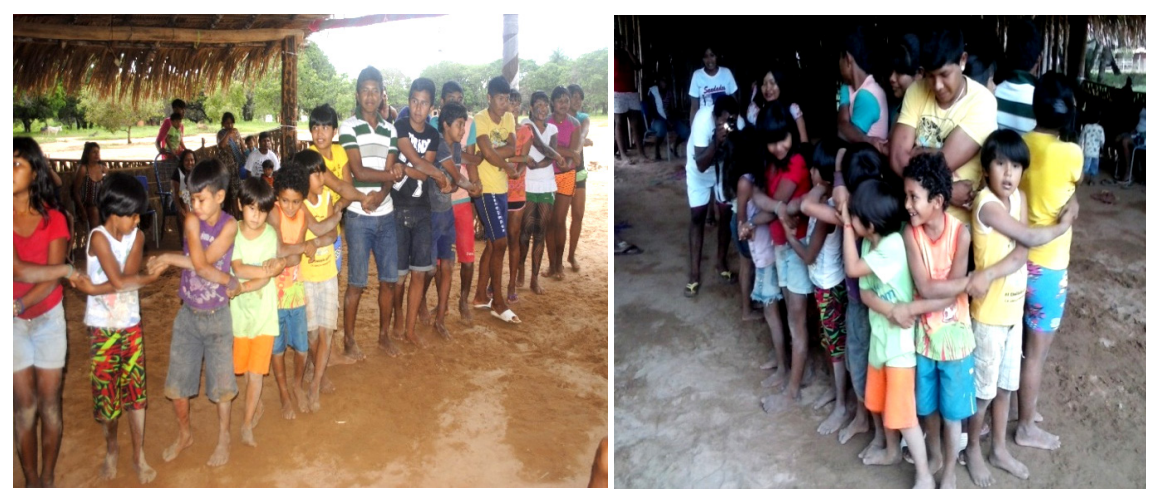

Fonte: As autoras, março de 2017.

Com o objetivo de garantir a compreensão do que seja a brincadeira A'cruu'te, apresentamos a seguir uma descrição na língua gavião: A'cruu'tejýycronnycohteharyrquica me hapyyryyte, ne me ajpenjõ' cry'nyhowaheh,quicapohmjôhmacpohnyxyny, quica me to, me 'crycrehapuxtomo, quicaêh 'protpime 'cohneamjõ hjapy,quicaêh 'protpijôhmtomejêhjpoh'nyajxii ne tome'coopohtomo ne me toajrenpaato ne caacpy'mytomecpu,pin quicajôhm,jôhmjomyxcry ne cawaaamrery'my, ho ne a'cruu'teny me hyycron te haryr.

A aula sobre a brincadeira de $A$ 'cruu 'te foi expositiva e prática, também no formato de oficina, e especificamente oferecida para as crianças da Educação Infantil. Foram duas anciãs convidadas para mediar a atividade e houve ampla participação dos jovens estudantes do noturno e de toda a comunidade da Aldeia Riachinho, isso porque a compreensão de todos é a de que "a brincadeira é livre". Durante toda a tarde aprendemos sobre socialização divertida e inclusiva, mas aprendemos muito sobre união e a importância da amizade. A brincadeira $A$ 'cruu'te estava um pouco esquecida e no final do dia o sentimento que prevalecia era o de felicidade e orgulho por pertencer ao Povo Gavião - Pyhcopcati ji que reproduz a vida com alegria e amizade.

Acerca da alegria, não há como desprezar as reflexões empreendidas por Paulo Freire (2002, p. 80) quando o mesmo realça que a alegria é necessária à atividade educativa. Considerando que, por meio das brincadeiras é possível se ensinar cultura, entendemos também que a brincadeira pode ser compreendida com uma 
atividade educativa e, nesta atividade específica, a alegria sempre se faz presente. Neste sentido, o ambiente que se instaura é de imensa criatividade e fortalecimento cultural.

Nabrincadeira Pýr Cahýc, a terceira sobre a qual discorreremos aqui, também há a necessidade de as pessoas envolvidas fazerem uma grande fila. Logo em seguida todos os participantes devem deitar no chão, sempre de frente. Assim sendo, todas as pessoas ficam deitadas com a barriga no chão e enfileiradas lado a lado. Organizadas desta forma, a pessoas que estiver em uma das extremidades da fila dá início à brincadeira quando começar a rolar por cima das demais pessoas. Ela vai se enrolando até chegar na outra extremidade da fila, quando se deitará de barriga para baixo alongando a fila. No mesmo instante, aquela que seria a segunda pessoa da ponta oposta de onde essa se deitou, começa a fazer o mesmo movimento. Assim a brincadeira segue sucessivamente, uma pessoa após outra, sendo que todas rolarão umas por cima das outras, em um contínuo que não tem hora para acabar.

Fotos 6 e 7 - Brincadeira Pýr Cahýc.
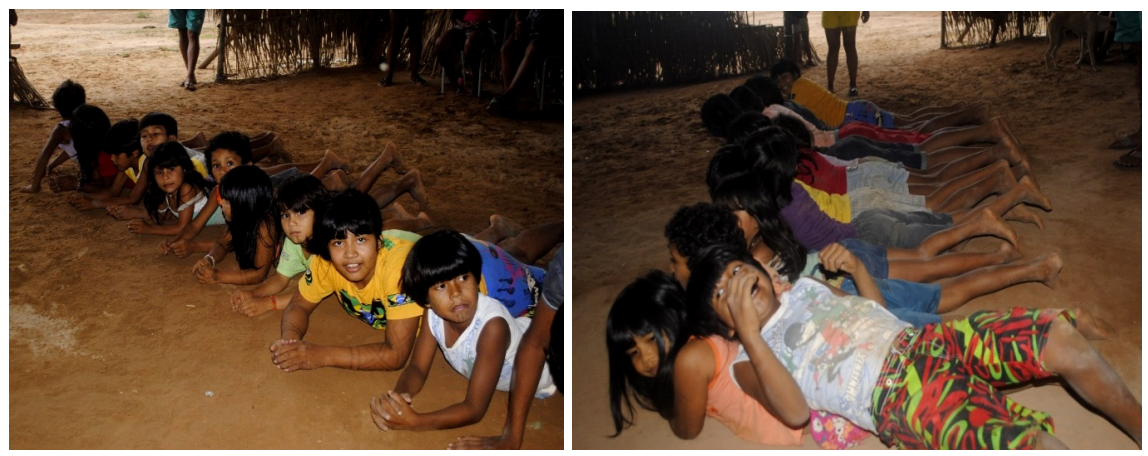

Fonte: As autoras, março de 2017.

$\mathrm{Na}$ busca por uma abrangência maior da explicação desta brincadeira, apresentamos a seguir uma descrição da brincadeira A'cruu te em língua gavião: Pýr Cahýc jýycronnycohtehajyrquica me hapyyryy te ne me hoopruupo ne me hêe-cwah, quicajôhma 'poh 'nyno na quica me hêepohno ne cahamohamjôhtoêhjpeexirtomo ne êhjpecamentomo ne caho'cu'nyno,quicajôhmtow ne me tohajyrtomo ne ca me tohêecohw, ne Pýr Cahýcny me hyycron te hajýr. 
Com a realização dessa aula sobre a brincadeira de Pýr Cahýc aprendemos mais uma vez que o conhecimento deve acontecer de maneira livre, inclusive fora do espaço escolar; de maneira participativa, não se restringindo ao quadro docente e discente; e de maneira responsável, assegurando o respeito aos elementos da cultura na qual os indivíduos estão inseridos. É a partir da vivência, interação sociocultural e reciprocidade que os conhecimentos se movimentam e os saberes se expandem. Com a brincadeira Pýr Cahýc, a alegria de pertencer a um povo que produz a felicidade cotidianamente foi renovada.

No que se refere à reciprocidade, que é amplamente trabalhada, destacamos que as trocas simbólicas marcam presença durante esta e as outras brincadeiras. Noções como "dar, receber e retribuir" produzem uma simpatia entre os membros do grupo, instaurando laços de união e semelhança (DIAS, 2011). Neste sentido, a reciprocidade e a solidariedade estruturam um sistema de trocas simbólicas com raízes fortemente assentadas na cultura que, no caso específico, são transmitidos e revitalizados pelas brincadeiras que são ensinadas e aprendidas.

A quarta brincadeira aqui estudada é a Pryyhre 'proxý, na qual os participantes devem fazer uma fila para que se inicie a diversão. A postura corporal na Pryyhre'proxý deve ser com os pés e mãos apoiados no chão, arquear as costas, elevando-as o mais alto que conseguir. E todas as pessoas da fila lateral imitam um túnel por onde uma das pessoas participantes que é escolhida para representar o tatu vai passar. Finalizada sua passagem é chegada a vez do seguinte da fila e assim sucessivamente. Se a pessoa que está representando o tatu esbarrar naquelas que estão representando o túnel, todos caem por cima do tatu que fica preso e a brincadeira acaba. A queda de todos em cima do tatu é um momento de muitas risadas e não indica o fim da diversão já que a Pryyhre 'proxý pode recomeçar e se repetir quantas vezes o grupo quiser. 
Fotos 8 e 9 - Brincadeira Pryyhre'proxý.
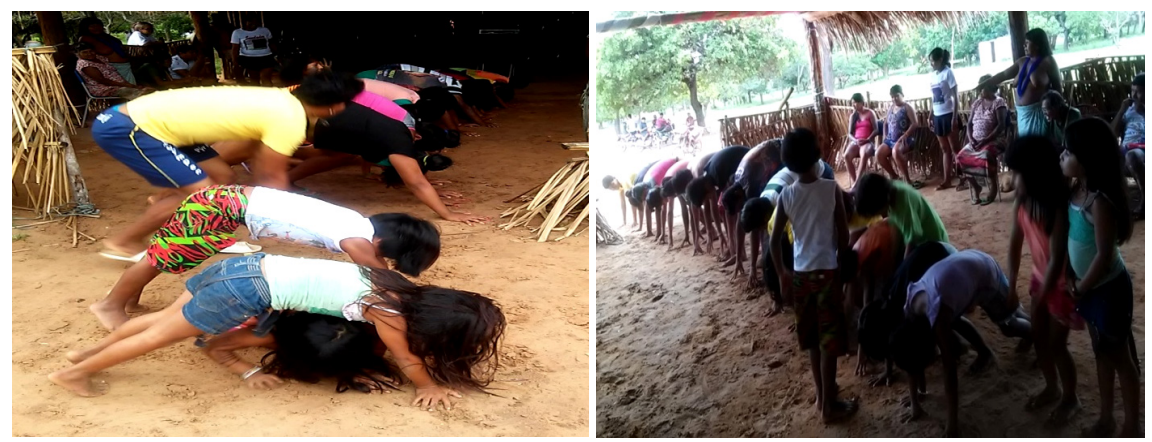

Fonte: As autoras, março de 2017.

Para que um leitor ou leitora gavião compreenda mais detalhadamente esta brincadeira, apresentamos a seguir uma descrição da mesma em língua gavião: Mohndehny me jýycron te hajýr,quica me hêehoo ne me hapýyryyre te pequicaajxyjôhmpitun ne jôhmme'crycrehapuxto te,ne came'cohneêhmpraapa,quicaho'cu'nytaquicajôhmhecquicamohndehhêepohêh 'pym, quica ne ryymy,jôhmhecnoorequicaho'cu'nyêhntaawyrcato,nemohndehjýycronnycohtehajýr.

A brincadeira de Pryyhre'proxý também ocupou toda a tarde no formato de oficina. O ponto central desta brincadeira é o cuidado que os participantes devem ter para não tocarem a outra pessoa e não derrubarem os outros colegas. Noções de espaço e limites do próprio corpo são aprimoradas com a Pryyhre'proxý. No momento de organização da brincadeira há todo um cuidado para orientar os pequenos a passarem por baixo dos maiores. Esses pequenos representam o tatu e os maiores representam uma espécie de armadilha que não pode ser tocada, sob pena de ser disparada. A diversão é garantida e também são despertadas noções relacionadas ao cuidado e à percepção do perigo por se fazer presente. A cultura gavião é fortalecida em instantes que são de ensino e aprendizagem para todos.

A brincadeira de Cahowcra'crat, a quinta trabalhada neste artigo, conta também com a participação das crianças, adolescentes e adultos e todos quantos quiserem brincar. A Cahowcra'crat consiste basicamente em escolher uma pessoa para ser enterrada com areia. O participante selecionado senta-se e coloca a mão em cima do joelho, em seguida os colegas que estão participando da brincadeira 
começam a enterrar o escolhido até o umbigo. O diferencial nesta brincadeira é o diálogo que é instaurado. Alguém pergunta para a pessoa que está sendo enterrada:- Êhmpohtemyaapicruurejêecree?E a pessoa que está sendo enterrada responde:- Cahowcraa'cratte temyêhjpiêh'cree. Depois desta resposta, todas as pessoas falam: Jõ'pji, jó 'pji,pji. Ao mesmo tempo em que enunciam começam a retirar rapidamente com as mãos toda a areia que cobria até o umbigo. $\mathrm{O}$ objetivo édesenterrar rapidamente para encerrar a brincadeira.

Fotos 10 e 11 - Brincadeira Cahowcra'crat.
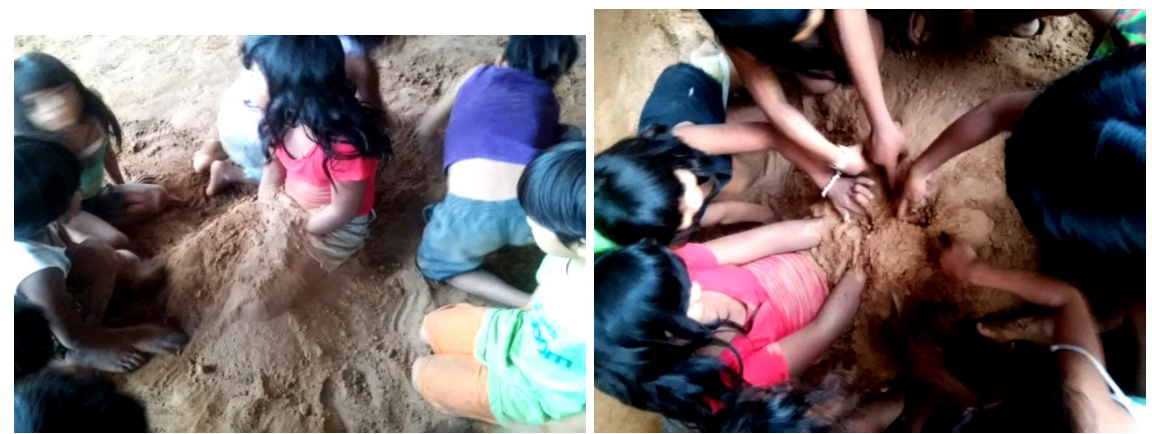

Fonte: As autoras, março de 2017.

A Cahowcra'crat segue a seguir explicada detalhadamente na língua gavião para que a leitura deste artigo chegue também àquelas pessoas que falam gavião: Cahowcraa'cratjýycron te hajýr, quica me jôhmtoamjõhmmyawcapêhpequicajôhmêh'cõhncrynyjyh ne amjõcji'cymho'craaxeh,ne ca me jôhmjýyme ne me hyymertohôntotnyhetep,pequicajôhmêh'cohquej ne cacohmy,êhmpoh te myaapicruurejêecree,quicohmyCahowcraa 'cratti 'temyêhjpiêh 'cree,quicame'cohnetojõ 'pji,jõ 'pji,jó'pji ne ca me cohpryytoawjaripequicajôhmtow ne ca me tohecownymquicamecmyêh 'tyxhyycronprymquica me acrepiimyacroh ne Cahowcraa'cratjýycron te hajýr.

Para realizar essa brincadeira é necessário usar muita areia onde uma criança que é escolhida será coberta com areia, enquanto estiver sentada com as mãos no joelho, até o umbigo. A Cahowcra 'crat é uma brincadeira rápida, com duração de cerca de 5 minutos e que pode ser repetida quantas vezes os participantes quiserem. Quase esquecida a Cahowcra'crat foi revitalizada durante 
as aulas do estágio supervisionado. Ao mesmo tempo, também foram revitalizadas concepções que fortalecem a importância do diálogo e da comunicação entre os Pyhcopcati ji.

\section{Algumas considerações}

Consideramos que o aprendizado acumulado foi intenso na experiência do estágio supervisionado, desde o primeiro aprendizado que passa pela concepção de que as possibilidades de sociabilidade e interação sociocultural, por meio de brincadeiras, entre o Povo Gavião - Pyhcopcati ji, são múltiplas e complexas, até a compreensão de que as brincadeiras são importantes espaços de produção, reprodução e revitalização da cultura. Os espaços educacionais estimulam processos de significação, o que, por sua vez, evidencia a cultura como uma entidade coletiva. Essa dimensão foi demonstrada sobremaneira na participação e envolvimento de todos os sujeitos - crianças, adolescentes, adultos e anciãos, estudantes - ou não nesses processos de significação.

A partir da realização das aulas, no âmbito do estágio supervisionado, o "orgulho de ser Pyhcopcati ji" pôde ser notado em instantes nos quais se ensina e se aprende, brincando, sobre o necessário convívio harmônico e respeitoso entre todos os seres vivos; sobre união entre todas as pessoas e a importância da amizade; sobre a importância da convivência, interação sociocultural e reciprocidade que movimentam conhecimentos e expandem saberes; sobre noções relacionadas ao cuidado, auto-cuidado e à percepção do perigo; e, sobre a necessária revitalização de concepções que destacam a importância do diálogo, da comunicação entre os Pyhcopcati ji. Enfim, são elementos de cultura que são transmitidos, valorizados e revitalizados em um ambiente de descontração e diversão.

Nos contextos de brincadeiras, um ingrediente que sempre se faz presente é a alegria. Sobre a alegria consideramos, assim como o faz Paulo Freire (2002), que a mesma conta com a esperança como forte aliada, também sempre presente em contextos de brincadeiras. Onde há esperança, a alegria não encontra obstáculos e em contextos assim consolidados o aprender e o ensinar fluem livremente e de maneira respeitosa às multiplicidades do existir. Regada com alegria, a transmissão cultural é garantida na atividade de brincar, já que a 
brincadeira é estruturada de acordo com os sistemas de significação cultural do grupo ao qual os participantes da brincadeira pertencem.

Cỹh, xỳmpe. Fim.

\section{Referências}

BROUGÉRE, Gilles. Brinquedo e Cultura. $4^{\mathrm{a}}$ ed. São Paulo: Cortez, 2001.

DIAS, Luciana de Oliveira (Org.). Pyhcop Cati Ji JõPji: Território Gavião - MA. Goiânia - GO: Editora da Imprensa Universitária, 2017.

DIAS, Luciana de Oliveira. "Dar, Receber e Retribuir": a dádiva enquanto princípio de sociabilidade. In: LUCENA, Andréa Freire; et al (Orgs.). Cooperação e Inclusão Social. Goiânia: Editora da PUC Goiás, 2011.

FREIRE, Paulo. Pedagogia da Autonomia: saberes necessários à prática educativa. $22^{\mathrm{a}}$ ed. Rio de Janeiro: Paz e Terra, 2002.

GEERTZ, Clifford. A Interpretação das Culturas. Rio de Janeiro: Zahar Editores, 1989.

GÓES, Maria Cecília R. As relações intersubjetivas na construção de conhecimentos. In: GÓES, Maria Cecília R.; SMOLKA, Ana Luiza B. (Orgs.). A significação nos espaços educacionais: interação social e subjetivação. Campinas: Papirus, 1997. p. 11-28.

LARAIA, Roque de Barros. Cultura: Um Conceito Antropológico. RJ: Zahar, 1986.

Recebido para publicação em fevereiro de 2018. Aceito para publicação em maio de 2018. 